\title{
Long-Term Results of Radical Surgery and Adjuvant Radiotherapy in a Case of Primary Malignant Fibrous Histiocytoma of the Kidney
} \author{
Vito Mancini ${ }^{1}$, Pantaleo Bufo ${ }^{2}$ and Giuseppe Carrieri ${ }^{1}$ \\ ${ }^{I}$ Department of Urology and Renal Transplantation, University of Foggia, Foggia, Italy \\ ${ }^{2}$ Department of Pathology, University of Foggia, Foggia, Italy
}

Luigi Cormio ${ }^{*}, 1$, Francesca Sanguedolce ${ }^{2}$, Paolo Massenio ${ }^{1}$, Giuseppe Di Fino ${ }^{1}$, Giuseppe Lucarelli ${ }^{1}$,

\begin{abstract}
Malignant fibrous histiocytoma of the kidney is an extremely rare tumor that is thought to arise from the renal capsule. Radical nephrectomy is the standard treatment for localized disease but $25 \%$ of patients die of metastatic disease despite radical surgical resection. In the absence of clear pathogenetic information, adjuvant treatment remains empiric. Herein, we describe the case of a previously healthy 58-year-old woman who underwent radical nephrectomy because of $8-\mathrm{cm}$ solid lesion of the right kidney; pathology revealed a pleomorphic malignant fibrous histiocytoma invading the perinephric fat, stage pT3aN0. She received adjuvant radiotherapy. To date, five years after surgery, she is alive and there is no evidence of disease. To our knowledge, this is the first report of favorable long-term results of surgery and adjuvant radiotherapy in a locally-advanced malignant fibrous histiocytoma of the kidney.
\end{abstract}

Keywords: Adjuvant radiotherapy, histiocytoma, kidney, long-term, radical surgery, rare tumor.

\section{INTRODUCTION}

Malignant fibrous histiocytoma (MFH) is an extremely rare tumor that belongs to a heterogeneous group of soft tissue malignancies featuring high pleomorphic cells in a haphazard arrangement. MFH of the kidney is thought to arise from the renal capsule but it is not clear yet whether or not it is a non-epithelial renal tumor. As a matter of fact, genetic studies have shown that MFH cells, rather than being histiocytic, show strict analogies with bone-marrow derived mesenchymal stem cells [1], which are currently regarded as the putative progenitor cells. Moreover, lack of a distinct gene cluster for this type of tumor suggests that most MFHs should be considered pleomorphic variants of different renal sarcomas, specifically lipo- and leiomyo- sarcomas $[2,3]$. Radical surgery represents the mainstay of MFH treatment; nevertheless, $25 \%$ of patients with localized disease die within 1 year despite radical surgical resection. Lack of clear pathogenetic information makes empiric any adjuvant treatment, thus often unsuccessful. Herein, we report for the first time the long-term results of a pT3a MFH treated with radical surgery and adjuvant radiotherapy, and discuss challenges in diagnosis and treatment of this uncommon neoplasm.

\section{CASE REPORT}

In a previous paper we reported a case of a 68 -year-old with a diagnosis of a renal malignant fibrous histiocytoma

*Address correspondence to this author at the Department of Urology and Renal Transplantation, University of Foggia, Viale Luigi Pinto 1, 71121 Foggia, Italy; Tel: +393208584073; E-mail: luigicormio@libero.it
[4]. In this report we summarize the clinical and pathological findings with an updated immunoistochemical staining for different markers and provide data about the long-term outcome of this unusual neoplasia. A previously healthy woman presented with a one-month history of evening fever; there were no haematuria nor abdominal or flank pain. Laboratory analysis showed normal renal function, mild anemia (hemoglobin: $9.9 \mathrm{~g} / \mathrm{dl}$ and hematocrit: 32\%), leukocytosis with normal lymphocyte count $\left(5.1 \times 10^{3} / \mu 1\right)$, increased erythrocyte sedimentation rate $(88 \mathrm{~mm} / \mathrm{hr})$, along with increased alpha-1 (4,94\%) and alpha-2 (16.82\%) and decreased gamma $(10.3 \%)$ globulins. There were no abnormalities on plain chest X-ray. Abdominal ultrasonography (US) showed a roundish hypo-echoic 8-cm solid lesion in the lower pole of the right kidney. Computed tomography (CT) confirmed the presence of an $8-\mathrm{cm}$ solid mass with heterogeneous enhancement; no lymphadenopathy was noted. Right radical nephrectomy with hilar lymph node dissection was performed for a suspicious renal cell carcinoma (RCC) of the right kidney. Postoperative course was uneventful and the patient was discharged on $4^{\text {th }}$ postoperative day. Macroscopic examination of the resected specimen revealed a solid grayish lesion, which microscopically consisted of haphazardly arranged highly atypical to pleomorphic, sometimes multinucleated cells, admixed with scattered inflammatory cells, with a brisk mitotic activity. Immunohistochemical analysis revealed intense vimentin staining, and negativity for cytokeratins, S100 protein, desmin, CD34, smooth muscle actin and lymphoid lineage markers in neoplastic cells (Fig. 1). On the basis of morphologic and immunophenotypic findings, a diagnosis of pleomorphic malignant fibrous histiocytoma (MFH) invading the perinephric fat, stage pT3aN0, was 
established. The patient received adjuvant radiotherapy (50 Gy in 25 sessions), which was well tolerated, and the patient was scheduled for a strict follow-up program including chest-abdomen-pelvis computed tomography (CT) scanning every six months and a bone scan every year. To date, 72 months after surgery, the patient is alive with no evidence of disease recurrence.
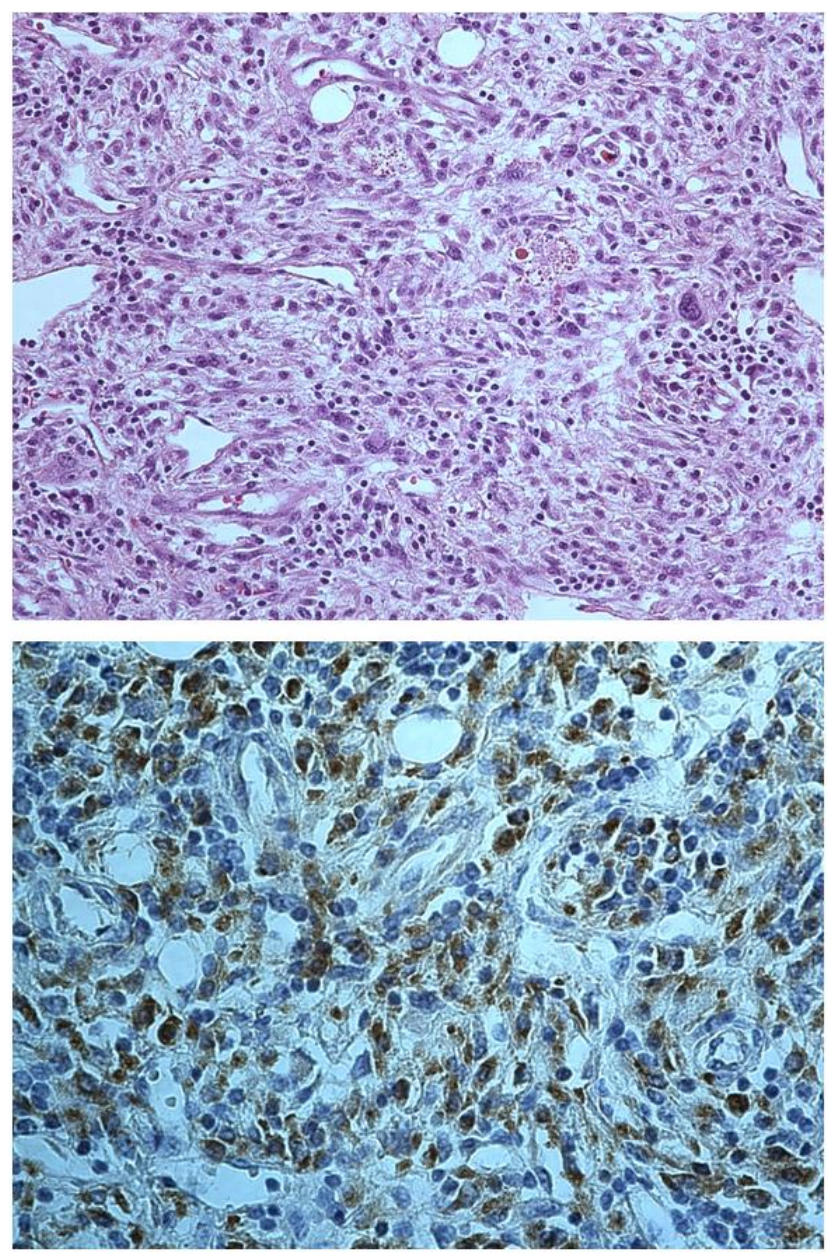

Fig. (1). Pleomorphic sarcoma (top) with highly atypical cells admixed with inflammatory cells (haematoxylin-eosin, original magnification 200X); neoplastic cells (bottom) show intense staining for vimentin (original magnification 400X).

\section{DISCUSSION}

Renal MFH is an extremely rare tumor with heterogeneous clinical features. Based on the few reported cases, it may present with abdominal or flank pain, abdominal mass, fatigue, unexplained fever, and even acute urinary retention [5-8]. Routine investigations may reveal anemia and/or leukocytosis with neutrophylia [5]. MFH has no specific US patterns; the diagnostic yield of CT scan and magnetic resonance imaging (MRI) for MFH seems to be related to the amount of fibrous tissue, the degree of vascularization, and the cellularity of the tumour [9]. The presence of a mass that appears hypointense on T2-weighted images and displays calcifications has been reported to be highly suggestive for MFH [7,9]. Nevertheless, almost all cases mimic RCC and are treated accordingly. In the absence of distant metastases, treatment involves radical nephrectomy. The diagnosis of MFH relies heavily on pathologic evaluation. MFH features high pleomorphic cells in a haphazard arrangement, with several possible patterns and variants, including storiform-pleomorphic, myxoid, giant cell, inflammatory, angiomatoid [10]. Such cells usually show intense positivity for vimentin, but negativity for cytokeratin, S-100 protein, and lymphoid lineage markers; this panel of immunohistochemical markers plays a pivotal role in the differential diagnosis with sarcomatoid carcinoma. Radical surgical resection is of major importance as local recurrence is associated with poor prognosis even in case of early diagnosis and treatment [11]. Adjuvant radiotherapy or chemotherapy, recommended to reduce the risk of cancer-related death, have yielded conflicting results $[5-7,12,13]$. As mentioned above, molecular studies suggest that most MFHs should be considered pleomorphic variants of different sarcomas. Accordingly, our patient underwent to adjuvant radiotherapy for the non-invasive nature, the low risk of side effects, and the responsiveness of sarcomas to this type of treatment. Moreover, we were dealing with a locally-advanced disease with an aggressive behavior. Patients presenting with metastatic disease are extremely challenging. Percutaneous tumor biopsy usually is of little diagnostic value, thus radical nephrectomy, if possible, is mandatory. Various sarcoma-directed radio- and/or chemotherapy treatments have been used with variable results. Recently, tyrosine kinase inhibitors (TKIs) - which have been shown to be active in metastatic RCC - have been reported to provide beneficial effects also in treating metastatic renal MFH $[14,15]$. In conclusion, renal MFH is a rare and complex entity mimicking RCC. Treatment of localized and locally-advanced disease relies on radical surgical excision. Since renal MFH belongs to renal sarcomas, adjuvant radiotherapy should have the potential to reduce the risk of local recurrence. The reported case provides evidence for favorable long-term results of adjuvant radiotherapy; however, whether it definitely improves survival remains unknown. TKIs seem to represent new efficient opportunities to treat metastatic disease but molecular identification of targets amenable to specific therapy would be extremely welcome in the management of this rare but insidious disease.

\section{CONFLICT OF INTEREST}

The authors confirm that this article content has no conflict of interest.

\section{ACKNOWLEDGEMENT}

Declared none.

\section{REFERENCES}

[1] Gazziola C, Cordani N, Wasserman B, Carta S, Colombatti A, Perris R. Malignant fibrous histiocytoma: a proposed cellular origin and identification of its characterizing gene transcripts. Int J Oncol 2003; 23: 343-51.

[2] Nakayama R, Nemoto T, Takahashi H, et al. Gene expression analysis of soft tissue sarcomas: characterization and reclassification of malignant fibrous histiocytoma. Mod Pathol 2007; 20: 749-59. 
[3] Tschoep K, Kohlmann A, Schlemmer M et al. Gene expression profiling in sarcomas. Crit Rev Oncol Hematol 2007; 63: 111-24.

[4] Marchese R, Bufo P, Carrieri G et al. Malignant fibrous histiocytoma of the kidney treated with nephrectomy and adjuvant radiotherapy: a case report. Medicine 2010; doi: 10.1155/2010/ 802026

[5] Ghosh A, Dwivedi US, Kumar A. Inflammatory malignant fibrous histiocytoma of kidney: A case report. Pathol Res Pract 2008; 204: 857-61.

[6] Gupta R, Gupta S, Aggarwal D, Singh S. Primary pleomorphic undifferentiated sarcoma of kidney: A rare renal tumor. Indian $\mathrm{J}$ Pathol Microbiol 2008; 51: 573-6.

[7] Kitajima K, Morita YK, Okuda Y, Sugimura K. Malignant fibrous histiocytoma arising from the renal capsule. Magn Reson Med Sci 2003; 2: 199-202.

[8] Singh SK, Mandal AK, Agarwal MM, Das A. Primary renal inflammatory malignant fibrous histiocytoma: A diagnostic challenge. Int J Urol 2006; 13: 1000-2.

[9] Kwak HS, Kim CS, Lee JM. MR findings of renal malignant fibrous histiocytoma. Eur Radiol 2003; 13: L245-L246.
[10] Al-Agha OM, Igbokwe AA. Malignant fibrous histiocytoma between the past and the present. Arch Pathol Lab Med 2008; 132: 1030-5.

[11] May M, Marusch F, Helke C, Gunia S, Gastinger I, Hoschke B. Das lokal rezidivierende maligne fibröse Histiozytom der Niere oder des Samenstrangs. Urologe [A] 2004; 43: 976-81.

[12] Eroğlu M, Bakirtaş H, Cimentepe E, Unsal A, Ataoğlu O, Balbay MD. Malignant fibrous histiocytoma arising from the renal capsule. Urol Int 2005; 75: 368-70.

[13] Kim SJ, Ahn BC, Kim SR, et al. Primary malignant fibrous histiocytoma of the kidney. Yonsei Med J 2002; 43: 399-402.

[14] Mauri D, Panou C, Valachis A, Kamposioras K, Tsali L. Tyrosine kinase inhibitors in treatment of fibrous histiocytoma. Exp Oncol 2009; 31: 60-1.

[15] Eichelberg C, Heuer R, Chun FK, et al. Sequential use of the tyrosine kinase inhibitors sorafenib and sunitinib in metastatic renal cell carcinoma: a retrospective outcome analysis. Eur Urol 2008; 54: $1373-8$.

(C) Cormio et al.; Licensee Bentham Open.

This is an open access article licensed under the terms of the Creative Commons Attribution Non-Commercial License (http://creativecommons.org/licenses/by-nc/3.0/) which permits unrestricted, non-commercial use, distribution and reproduction in any medium, provided the work is properly cited. 